\title{
Sangramento Maciço em Cirurgia Onco-Ginecológica - Uso do Pack Hemostático
}

\author{
Massive Hemorrhage in Gynecologic Cancer Surgery - the Use of Hemostatic PACK \\ Heitor Ricardo Cosiski Marana, Jurandyr Moreira de Andrade \\ Ângelo do Carmo da Silva Matthes, Francisco Cyro Reis Campos do Prado Filho \\ Francisco Jose Cândido dos Reis, Sérgio Bighetti
}

\section{RESUMO}

\begin{abstract}
Objetivo: descrever o uso do pack hemostático, um tamponamento feito com compressas amarradas contra a superficie sangrante, que permanece in situ por 48 a 72 horas e que é uma das alternativas terapêuticas diante de quadro de sangramento maciço.

Pacientes e Métodos: avaliamos 3 casos de sangramento maciço durante o ato cirúrgico, com troca de 1,4 volemias em média $(1,2-2,4)$. Os parâmetros avaliados foram a efetividade hemostática do pack e alguns parâmetros das pacientes, como idade, quantidade e tipo de volume infundidos.

Resultados: a idade média destas pacientes foi de 57 anos (51, 56 e 64). Apenas uma paciente havia sido irradiada previamente. Os sangramentos foram de origem venosa, provenientes da fossa do nervo obturador, plexo iliaco e pré-sacral. Expansor volumétrico mais utilizado foi o SF 0,9\%, seguido dos hemoderivados e depois pelo Ringer lactato. Durante o ato cirúrgico duas pacientes foram submetidas a ligadura da hipogástrica, sem melhora do quadro. O uso de material sintético hemostasiante foi ineficiente nos três casos. Em duas pacientes a sutura com fio inabsorvivel da área sangrante apresentou considerável diminuição do sangramento, sem eliminá-lo completamente. Uma paciente faleceu antes de 24 horas com quadro de falência cardiaca. As outras duas pacientes desenvolveram insuficiencia renal aguda, e uma, pneumonia aspirativa durante a re-exploração cirúrgica.

Conclusões: o quadro de sangramento maciço está relacionado a uma alta morbimortalidade. Dentre as medidas emergenciais de hemostasia o pack parece ser o mais adequado.
\end{abstract}

PALAVRAS-CHAVE: Cirurgia: complicações. Transfusão sangüinea. Choque hemorrágico.

\section{Introdução}

O sangramento maciço durante uma cirurgia pélvica é um quadro pouco freqüente, porém está associado a uma morbidade muito elevada, sendo potencialmente fatal ${ }^{4}$. Este quadro é mais comum

Departamento de Ginecologia e Obstetrícia da Faculdade de Medicina de Ribeirão Preto da Universidade de São Paulo. Correspondência:

Heitor Ricardo Cosiski Marana

Departamento de Ginecologia e Obstetrícia, Faculdade de Medicina de Ribeirão Preto -USP

Avenida Bandeirantes 3900, Campus da USP, Hospital das Clinicas, $8^{\circ}$ andar.

14.049-900 - Ribeirão Preto - SP

Tel (16) 633-0216, Fax (16) 633-0946 em cirurgias pélvicas extensas, como as cirurgias radicais e ultra-radicais, e em pacientes previamente irradiadas ${ }^{2}$. As primeiras medidas a serem tomadas são a identificação do vaso sangrante, seguida de pinçamento e amarração. A ligadura das hipogástricas e a compressão direta sobre a área sangrante são os passos seguintes. $\mathrm{O}$ uso de materiais sintéticos absorviveis em forma de esponja-gelatina hemostática (Gelfoam) e colas de fibrina pode ser útil ${ }^{7}$. Na eventual persistência do sangramento, que na maioria das vezes é difuso, o uso do pack hemostático torna-se uma opção viável. Assim, o conhecimento da seqüência e da técnica de coerção de sangramentos maciços em cirurgias pélvicas torna-se de grande importância. Em face 
da escassa literatura sobre este assunto, revisamos os fatores de risco cirúrgico e a mortalidade e a morbidade em cirurgias pélvicas com sangramento maciço, associando o relato de nossa experiência nesta situação.

\section{Pacientes e Métodos}

Três pacientes submetidas a cirurgias pélvicas no Departamento de Ginecologia e Obstetrícia da Faculdade de Medicina de Ribeirão Preto, da Universidade de São Paulo, apresentaram sangramento maciço durante o intra-operatório, necessitando o uso do pack hemostático. Entre os critérios empregados para inclusão nesta série estão a necessidade de hemotransfusão com mais de 10 unidades e troca de pelo menos uma volemia (em nossa pacientes, 1,4 volemias em média, variando de 1,2-2,4) e hemorragia que não pudesse ser controlada com nenhuma das medidas prévias, isoladas ou em associação, partindo da ligadura do(s) vaso(s) sangrante(s), ligadura das ilíacas internas (hipogástricas), terapia local com derivados sintéticos hemostasiantes ou suturas amplas com fios inabsorviveis.

Os dados das pacientes provêm de revisão de prontuários médicos e incluem idade, parâmetros da hemorragia, procedimentos cirúrgicos e tratamentos prévios. Analisamos ainda os tipos de hemoderivados utilizados no intraoperatório e cuidados intensivos implementados na vigência do pack hemostático. Obtivemos ainda outros dados a respeito da condição pós-operatória e complicações.

A média de idade destas pacientes foi de 57 anos $(51,56$ e 64). Apenas uma paciente havia sido irradiada previamente. As cirurgias realizadas foram Wertheim-Meigs (2) e histerectomia (1). Os sangramentos foram de origem venosa, provenientes da fossa do nervo obturador e plexos ilíaco e pré-sacral. Expansor volumétrico mais utilizado foi o SF 0,9\%, seguido dos hemoderivados e depois pelo Ringer lactato.

Diante do quadro de hemorragia pélvica, o cirurgião seguiu os seguintes passos: pinçou e ligou o vaso ou os vasos da região sangrante e, em caso de falha deste primeiro passo, comprimiu-se a área sangrante por meio de compressas. O anestesista foi alertado da possibilidade de sangramento maciço, para que pudesse providenciar acesso venoso para alto fluxo e solicitar hemoderivados ao banco de sangue. A seguir foram ligadas as artérias iliacas internas bilateralmente. Quando não havia sido realizada, neste ponto foi feita a retirada da peça cirúrgica, mantendo-se os reparos das ligaduras laterais da cúpula vaginal com fio Vicryl zero. As condições gerais da paciente eram reavaliadas e reiniciadas as tentativas de hemostasia, agora com suturas abrangentes da área sangrante, realizadas com fio inabsorvivel 2-0 (nylon ou Prolene) e colocação de resina sintética tipo esponja-gelatina (Gelfoam).

A opção pelo uso do pack era feita quando as tentativas acima descritas falhavam. Para tal utilizaram-se em média quatro compressas grandes, umedecidas em soro ou solução desgermante (PVPI) colocadas contra a região sangrante e aí comprimidas para estancar o sangramento (Figura 1). Utilizando-se dos fios de reparo ancorados na cúpula vaginal, transfixavase com estes o ligamento de Cooper ou as aponeuroses abdominais, fazendo-se fixação do pack. A parede era fechada e a paciente encaminhada à Unidade de Terapia Intensiva (UTI), com antibioticoterapia, sondagem vesical de demora, monitorização constante da pressão arterial, pulso e pressão venosa central, e com sedação para prevenção de picos hipertensivos. A paciente permaneceu nesta condição por 48 a 72 horas a fim de que se pudesse restabelecer o equilíbrio hemodinâmico, com recuperação de fatores da coagulação e eletrólitos, principalmente o cálcio. Quando da reexploração, deve-se ter à disposição material para embolização arterial angiográfica, caso seja necessário, assim como "taxinhas" de cabeça larga para fixação contra a parede óssea para obtenção de hemostasia.

As compressas foram retiradas uma a uma cuidadosamente com a utilização de soro morno em abundância e aspiração contínua. Caso ocorresse novo sangramento fora do controle, poder-se-ia optar por um novo pack. Ao contrário, caso não houvesse mais sangramento na área de sangramento antiga, esta região era recoberta por resina hemostasiante (Gelfoam), e a parede abdominal suturada por planos. A paciente retornava à UTI, mantendo-se os mesmos cuidados prévios por mais dois dias.

\section{Resultados}

Em dois dos três casos o sangramento foi resolvido após 48 horas de tamponamento. Uma paciente faleceu antes de 24 horas com um quadro de falência cardiaca. Esta paciente havia apresentado parada cardiaca na sala cirúrgica, tendo sido reanimada com massagem transdiafragmática. Após a reversão deste quadro, permaneceu instável, pois era portadora de hipertensão arterial crônica com repercussões 
cardiacas da doença. As outras duas pacientes desenvolveram IRA leve e uma destas sofreu aspiração do conteúdo gástrico durante a reexploração cirúrgica. A terceira paciente apresentou quadro de celulite na incisão.

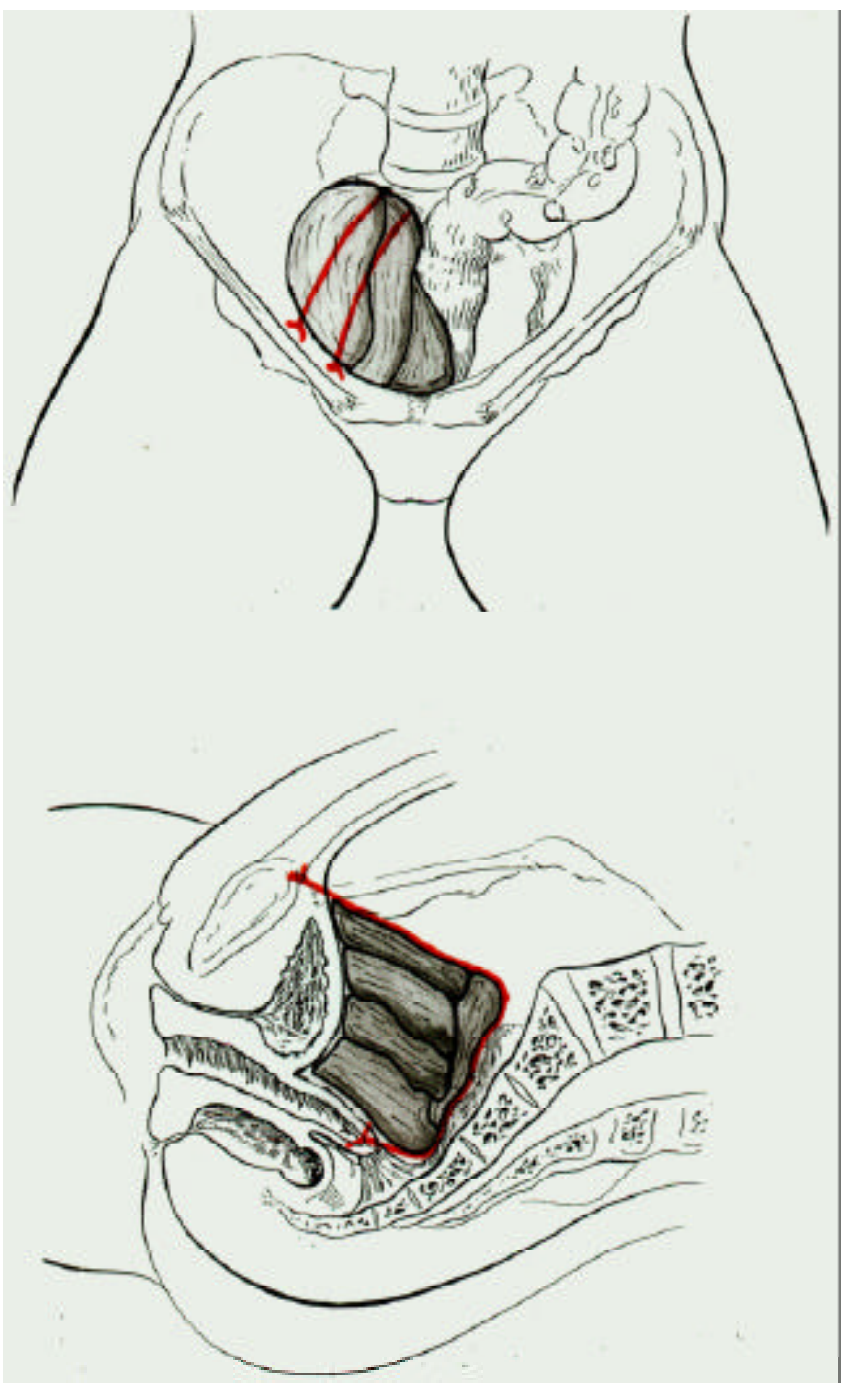

Figura 1 - Esquema de colocação das compressas embebidas em soro fisiológico contra a superfície sangrante e fixação com fios de sutura resistentes contra a cúpula vaginal posteriormente e contra aponeurose dos retos abdominais ou os ligamentos de Cooper anteriormente.

A remoção do pack, realizada em duas pacientes, não demonstrou a persistência de sangramento, após o que a região foi recoberta com resina hemostasiante e realizado o fechamento por planos.

\section{Discussão}

O uso do pack hemostático para as hemorragias intra-operatórias não é um procedimento novo. A primeira descrição de seu uso foi feita em 1908 para correção de sangramento hepático ${ }^{6}$. A primeira descrição desta técnica em cirurgia pélvica data de $1926^{3}$. Vários outros autores puderam relatar suas experiências pessoais ${ }^{1,2,4,7}$, porém a literatura nacional é pobre em detalhes de técnica, resultados e complicações.

O trauma cirúrgico da região da fossa do nervo obturador, plexos iliacos e pré-sacrais leva inevitavelmente a um sangramento profuso de dificil controle, principalmente pela existência de uma farta rede anastomótica nesta região. Outro fator importante é que o sistema venoso desta área não apresenta válvulas e goza de uma grande complacência, sendo um reservatório de sangue. Assim, na ocorrência de lesão vascular nestes plexos, o sangramento é abundante e de alta pressão hidrostática, com potencial capacidade para levar rapidamente ao choque ou à morte . $^{4}$

Muitas técnicas foram descritas para hemostasia em situações dramáticas como esta, incluindo-se a ligadura das hipogástricas, a embolização arterial angiográfica, colas de fibrina, resinas sintéticas e o pack hemostático ${ }^{5}$. Em nossa casuística o uso do pack pareceu-nos viável pela praticidade técnica e exeqüibilidade em diferentes situações, sem um grande aparato téc-nico, e está associado a cifras de morbimortalidade próprias aceitáveis.

A utilização do "pack" deve seguir uma monitorização da pressão distal, a fim de minorar o risco de compressão exagerada da veia cava inferior e seus ramos, que pode culminar em um quadro de falência renal aguda ${ }^{2,7}$. Duas de nossas pacientes apresentaram tal complicação e na literatura é a complicação grave mais freqüentemente relatada. Parece que a hipovolemia intraoperatória guarda uma estreita relação com esta complicação, que pode ser agravada pela utilização do pack.

Outra complicação potencial é a neuropatia tanto do obturador como dos ramos ciáticos, que pode persistir por até 6 meses após a cirurgia ${ }^{7}$. As complicações infecciosas são as mais freqüentes, podendo abranger desde uma celulite periincisional, até abscessos e sépsis, justificando assim a utilização de antibioticoterapia de largo espectro de ação ${ }^{2}$. Tivemos apenas um quadro de celulite na terceira paciente. O uso de antibióticos nefrotóxicos, no entanto, deve ser evitado.

As pacientes submetidas a cirurgias radicais após irradiação prévia são candidatas potenciais a complicação como a hemorragia maciça. O uso do pack hemostático está longe de ser a solução ideal, mas para a nossa realidade atual parece ser a melhor forma de enfrentar esta complicação. 


\section{SUMMARY}

Purpose: to introduce the use of the hemostatic pack, a tampon consisting of compresses tied to the bleeding surface and left in place for 48 to $72 \mathrm{~h}$ and which is one of the therapeutic alternatives to be used in this situation.

Patients and Methods: we evaluated 3 cases of massive bleeding during surgery, with the exchange, on average, of 1.4 blood volumes (1.2-2.4), in terms of hemostatic effectiveness of the pack and of some patient parameters such as age and amount and type of volume infused.

Results: the mean age of the patients was 57 years (51, 56 and 64). Only one had been previously irradiated. The bleeding was of venous origin, from the fossa of the obturator nerve, the iliac plexus and the presacral plexus. The volume expander most often used was $0.9 \%$ physiological saline solution, followed by blood derivatives and by Ringer lactate. Two patients were submitted to ligation of the hypogastric vein during surgery, with no improvement. The use of hemostatic synthetic material was inefficient in all three cases. In one of the patients, the use of nonabsorbable sutures to close the bleeding area led to a considerable reduction of bleeding, but did not eliminate it completely. One patient died before $24 \mathrm{~h}$ had elapsed, with signs and symptoms of heart failure. The other two patients developed acute renal failure and one of them developed aspirative pneumonia during surgical reexploration.

Conclusion: massive bleeding is related to high morbidity and mortality. Among the emergency measures used for hemostasis, the pack seems to be the most adequate.

KEY WORDS: Massive hemorrhage. Surgery: complications.

\section{Referências}

1. Cassels JW Jr, Greenberg H, Otterson WN. Pelvic tamponade in puerperal hemorrhage: A case report. J Reprod Med 1985; 30:689-92.

2. Finan MA, Fiorica JV, Hoffman MS, Barton DPJ, Gleeson N, Roberts WS, Cavanagh D. Massive pelvic hemorrhage during gynecologic cancer surgery: "Pack and go back". Gynecol Oncol 1996; 62: 390-5.

3. Logothetopulos K. Eine absolut sichere Blutstillungsmethode bei vaginalen und abdominalen gynäkologischen Operationen. Zentralbl Gynäkol 1926; 60: 3202-10.

4. Marsden DE, Cavanagh D. Hemorrhagic shock in the gynecologic patient. Clin Obstet Gynecol 1985; 28: $381-90$

5. O'Hanlan KA, Trambert J, Rodriguez-Rodriguez L, Goldberg GL, Runowicz CD. Arterial embolization in the management of abdominal and retroperitoneal hemorrhage. Gynecol Oncol 1989; 34: $131-5$.

6. Pringle JH. Notes on the arrest of hepatic hemorrhage due to trauma. Ann Surg 1908; 48: 541-9.

7. Stolfi VM, Milson JW, Lawery IC, Oakley JR, Church JM, Fazio VW. Newly designed occluder pin for presacral hemorrhage. Dis Colon Rectum 1992; 35: $166-9$ 